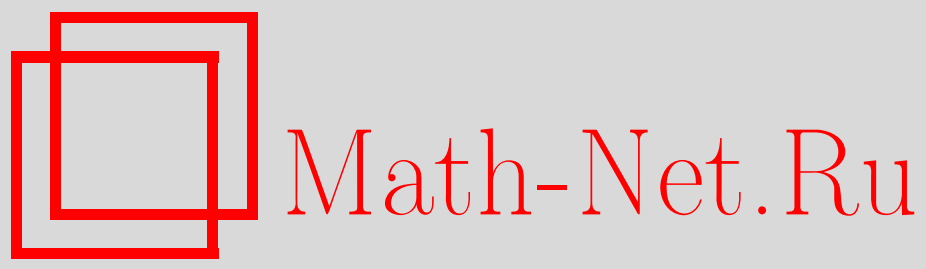

M. A. Arcones, The large deviation principle for stochastic processes. I, Теория вероятн. и ее примен., 2002, том 47, выпуск 4, 727-746

DOI: https://doi.org/10.4213/tvp3777

Использование Общероссийского математического портала MathNet.Ru подразумевает, что вы прочитали и согласны с пользовательским соглашением

http://www . mathnet.ru/rus/agreement

Параметры загрузки:

IP : 18.234 .156 .22

26 апреля 2023 г., 18:03:52

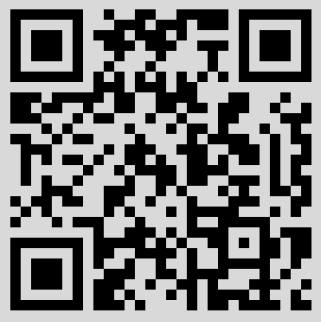


(C) $2002 \Gamma$.

ARCONES M. A.*

\title{
THE LARGE DEVIATION PRINCIPLE FOR STOCHASTIC PROCESSES. I
}

\begin{abstract}
Обсуждается принцип больших уклонений для случайных процессов как случайных элементов в $l_{\infty}(T)$. Показано, что принцип больших уклонений в $l_{\infty}(T)$ эквивалентен принципу больших уклонений для конечномерных распределений плюс условие экспоненциальной асимптотической равностепенной непрерывности относительно псевдометрики, которая превращает $T$ во вполне ограниченное псевдометрическое пространство. Этот результат позволяет получить необходимые и достаточные условия выполнения принципа больших уклонений для случайных процессов. В статье обсуждается принцип больших уклонений для различных типов случайных процессов.
\end{abstract}

Ключевые слова и фразы: большие уклонения, случайные процессы, гауссовские процессы, итерированное броуновское движение, процесс Пуассона.

1. The large deviation principle for stochastic processes. In many different situations, it is of interest to estimate the rate of convergence of certain probabilities. Often these probabilities converge exponentially fast. Several authors have considered large deviations and obtained different types of applications mainly to mathematical physics. General references on large deviations are [3], [33], [13], [12].

We study functional large deviations of stochastic processes following the approach to deal with measurability problems for the weak convergence of stochastic processes in [19]. We assume very little measurability restrictions and we use outer and inner probabilities. We refer to [31] and [15] for measurability considerations. We consider stochastic processes as random elements. By a random element, we mean a (nonnecessarily measurable) function from a probability space to an arbitrary set. We use the following definition of large deviation principle (LDP) for random elements.

$\mathrm{D}$ e f i n i t i o $\mathrm{n}$ 1.1. Given a sequence of random elements $\left\{X_{n}\right\}_{n=1}^{\infty}$ with values in a topological space $(S, \mathscr{T})$, a sequence of positive numbers $\left\{\varepsilon_{n}\right\}_{n=1}^{\infty}$ such that $\varepsilon_{n} \rightarrow 0$, and a function $I: S \rightarrow[0, \infty]$, it is said

* Department of Mathematical Sciences, Binghamton University, Binghamton, NY 13902-6000, U.S.A.; e-mail: arcones@math.binghamton.edu 
that $\left\{X_{n}\right\}$ satisfies the large deviation principle (LDP) with speed $\varepsilon_{n}^{-1}$ and with rate function $I$ if:

(i) For each $0 \leqslant c<\infty,\{z \in S: I(z) \leqslant c\}$ is a compact set;

(ii) for each set $A \subset S$,

$-I\left(A^{\circ}\right) \leqslant \liminf _{n \rightarrow \infty} \varepsilon_{n} \ln \left(\mathbf{P}_{*}\left\{X_{n} \in A\right\}\right) \leqslant \limsup _{n \rightarrow \infty} \varepsilon_{n} \ln \left(\mathbf{P}^{*}\left\{X_{n} \in A\right\}\right) \leqslant-I(\bar{A})$,

where for a set $B, I(B)=\inf \{I(x): x \in B\}$.

In the previous definition and in the future, we denote $\inf (\varnothing)=\infty$. Condition (i) in Definition 1.1 implies that $I$ is a lower semicontinuous function. The assumptions in Definition 1.1 imply that there exists a $z \in S$ such that $I(z)=0$. Typically, $z$ is unique and $X_{n} \stackrel{\mathrm{P}}{\rightarrow} z$. A function $I: S \rightarrow[0, \infty]$ is called a good rate function if condition (i) in Definition 1.1 holds.

We study the LDP for a sequence of stochastic processes $\left\{U_{n}(t): t \in T\right\}$ with values in a Banach space $B$, which are bounded with probability one, where $T$ is an index set. We consider $\left\{U_{n}(t): t \in T\right\}$ as a random element with values in the Banach space $l_{\infty}(T, B)$, the set of bounded functions in $T$ with values in $B$ with the norm $|z|_{\infty}:=\sup _{t \in T}|z(t)|_{B}$, where $|\cdot|_{B}$ denotes the norm in $B$. We do not assume that $\left\{U_{n}(t): t \in T\right\}$ is a random variable (r.v.) with values in $l_{\infty}(T, B)$ endowed with the Borel $\sigma$-field. We only assume that for each $t \in T, U_{n}(t)$ is a r.v.

D e f i n it i o n 1.2. Given a sequence of stochastic processes $\left\{U_{n}(t)\right.$ : $t \in T\}$ with values in a Banach space $B$, such that for $n$ large enough $\mathbf{P}_{*}\left\{\sup _{t \in T}\left|U_{n}(t)\right|_{B}<\infty\right\}=1$, a sequence of positive numbers $\left\{\varepsilon_{n}\right\}_{n=1}^{\infty}$ such that $\varepsilon_{n} \rightarrow 0$, and a function $I: l_{\infty}(T, B) \rightarrow[0, \infty]$, we say that $\left\{U_{n}(t): t \in T\right\}$ satisfies the LDP in $l_{\infty}(T, B)$ with speed $\varepsilon_{n}^{-1}$ and with rate function $I$ if:

(i) For each $0 \leqslant c<\infty,\left\{z \in l_{\infty}(T, B): I(z) \leqslant c\right\}$ is a compact set of $l_{\infty}(T, B)$;

(ii) for each set $A \subset l_{\infty}(T, B)$,

$$
\begin{aligned}
-I\left(A^{\circ}\right) & \leqslant \liminf _{n \rightarrow \infty} \varepsilon_{n} \ln \left(\mathbf{P}_{*}\left\{\left\{U_{n}(t): t \in T\right\} \in A\right\}\right) \\
& \leqslant \limsup _{n \rightarrow \infty} \varepsilon_{n} \ln \left(\mathbf{P}^{*}\left\{\left\{U_{n}(t): t \in T\right\} \in A\right\}\right) \leqslant-I(\bar{A}) .
\end{aligned}
$$

We denote $l_{\infty}(T)=l_{\infty}(T, \mathbf{R})$. It is easy to see that a sequence of stochastic processes $\left\{U_{n}(t): t \in T\right\}$ with values in a Banach space $B$ satisfies the LDP in $l_{\infty}(T, B)$ with speed $\varepsilon_{n}^{-1}$ if and only if the sequence of stochastic processes $\left\{V_{n}(t, f): t \in T, f \in B_{1}^{*}\right\}$ satisfies the LDP in $l_{\infty}\left(T \otimes B_{1}^{*}\right)$ with speed $\varepsilon_{n}^{-1}$, where $B_{1}^{*}$ is the unit ball of the dual of $B$ and $V_{n}(t, f)=f\left(U_{n}(t)\right)$, for $t \in T$ and $f \in B_{1}^{*}$. So, the study of the LDP for stochastic processes with values in a Banach space reduces to the study of the LDP for real valued stochastic processes. We will usually consider stochastic processes with values in $\mathbf{R}$. But, sometimes, we will need to consider multidimensional 
valued stochastic processes. We also have that a sequence of r.v.'s $\left\{X_{n}\right\}_{n=1}^{\infty}$ with values in a Banach space $B$ satisfies the LDP with speed $\varepsilon_{n}^{-1}$ if and only if the stochastic process $\left\{f\left(X_{n}\right): f \in B_{1}^{*}\right\}$ satisfies the LDP with speed $\varepsilon_{n}^{-1}$. So, our results give necessary and sufficient conditions for the LDP of Banach space valued r.v.'s (see Corollary 3.4).

It is well known that functional formulations of limit theorems have many different applications (see for example [31] and [15]). We will use the functional LDP to obtain the tail behavior of the iterated fractional Brownian motion. For stochastic processes whose paths are not bounded in $T$, but they are bounded in subsets of $T$, it is possible to obtain a LDP in other spaces (see Theorem 3.2).

In Section 2, we present an extension of the contraction principle. The contraction principle says that we may apply a continuous function to a sequence of random elements satisfying the LDP and still have the LDP for the transformed sequence. We extend this technique to not necessarily continuous functions. We will need this extended contraction principle because the composition of stochastic processes is not a continuous functional in $l_{\infty}(\mathbf{R}) \times l_{\infty}([0, M])$, where $M>0$.

In Section 3, we show that a sequence of bounded stochastic processes satisfies the LDP if and only if the finite dimensional distributions satisfy the LDP and an exponential asymptotic equicontinuity condition holds with respect to certain pseudometric which makes $T$ totally bounded. Some applications of this characterization are given. We see that the LDP in Definition 1.2 with $B=\mathbf{R}$ implies that $(T, \rho)$ is a totally bounded pseudometric space and an exponential asymptotic equicontinuity condition holds with respect to this pseudometric, where

$$
\begin{aligned}
\rho(s, t) & =\sum_{k=1}^{\infty} k^{-2} \min \left(\rho_{k}(s, t), 1\right), \\
\rho_{k}(s, t) & =\sup \left\{\left|u_{2}-u_{1}\right|: I_{s, t}\left(u_{1}, u_{2}\right) \leqslant k\right\}
\end{aligned}
$$

and $I_{s, t}$ is the rate function for the LDP for $\left(U_{n}(s), U_{n}(t)\right)$. An easy method (see Theorem 6.3 in Part II of the present paper) to prove that a sequence of stochastic processes $\left\{U_{n}(t): t \in T\right\}$ in $l_{\infty}(T)$ does not satisfy the LDP is to check that $\left(T, \rho_{k}\right)$ is not totally bounded. Several applications of this characterization of the LDP are given. We obtain necessary and sufficient conditions for the LDP for a sequence of r.v.'s with values in a separable Banach space. We obtain minimal sufficient conditions for the LDP for stochastic processes with either increasing or convex paths. We present minimal conditions for obtaining that the composition of two stochastic processes satisfies the LDP.

Several authors have studied the tightness in the LDP, in a similar way weak convergence is studied. Puhalskii [25] showed that if a sequence of 
r.v.'s with values in a metric space is exponential tight, then there exists a subsequence satisfying the large deviation principle. He also showed that for stochastic processes with values in $D[0, M]$ an exponential asymptotic equicontinuity condition implies tightness.

In Section 4 (see Part II), we give the form of the rate function for the LDP in $l_{\infty}(T)$ for many of the considered cases. We will that under certain conditions the rate function is given by

$$
I(z)=\inf \left\{\int \Psi(\gamma(x)) d \mu(x): \int f(x, t) \gamma(x) d \mu(x)=z(t) \text { for each } t \in T\right\},
$$

where $\Psi$ is a convex function, $\mu$ is a (positive) measure in a measurable space $(S, \mathscr{S})$ and $\{f(x, t): t \in T\}$ is a class of measurable functions. In the cases considered in this paper either $\Phi(x)=p^{-1}|x|^{p}$, for some $p>0$ or $\Phi(x)=e^{x}-1$. We also see that under certain conditions, the rate function in the LDP for some certain stochastic processes has the form

$$
I(z)= \begin{cases}\int_{0}^{M} \Psi\left(z^{\prime}(t)\right) d t, & \text { if } z(0)=0 \text { and } \\ \infty, & z \text { is absolutely continuous } \\ \text { otherwise. }\end{cases}
$$

We obtain the rate function of the composition of two stochastic processes, when the rate function of each of the considered stochastic processes has the form (1.3) with different functions $\Psi$.

In Section 5, we give necessary and sufficient conditions for the LDP for a sequence of Gaussian processes. Several applications to the iterated Brownian motion are presented. A Brownian motion $\{B(t): t \in \mathbf{R}\}$ is a centered Gaussian process with covariance

$$
\mathbf{E}[B(s) B(t)]=\min (|s|,|t|) I(s t \geqslant 0), \quad s, t \in \mathbf{R} .
$$

If $\left\{B_{1}(t): t \geqslant 0\right\}$ and $\left\{B_{2}(t): t \geqslant 0\right\}$ are two independent Brownian motions, then $\{B(t): t \in \mathbf{R}\}$ is a Brownian motion, where $B(t)=B_{1}(t)$ for $t \geqslant 0$, and $B(t)=B_{2}(-t)$ for $t \leqslant 0$. The process $\{B(B(t)): t \in \mathbf{R}\}$ is called an iterated Brownian motion. Some authors use the term «iterated Brownian process» for the process $\left\{B_{1}\left(B_{2}(t)\right): t \in \mathbf{R}\right\}$, where $\left\{B_{1}(t): t \in \mathbf{R}\right\}$ and $\left\{B_{2}(t): t \in \mathbf{R}\right\}$ are two independent Brownian motions. Funaki [18] used a modification of the iterated Brownian motion to give a probability solution to the partial differential equation

$$
\frac{\partial u}{\partial t}=\frac{1}{8} \frac{\partial^{4} u}{\partial x^{4}} \quad \text { with } \quad u(0, x)=u_{0}(x) .
$$

Deheuvels and Mason [11], Burdzy ([6], [7]), Arcones [1], Hu and Shi [20], Shi [29], Hu, Pierre-Loti-Viaud, and Shi [21], Csáki; Csörgö, Földes, and 
Révész [9], Khoshnevisan and Lewis ([22], [23]) and Csáki, Földes and Révész [10] have studied different properties of the iterated Brownian motion. We will prove that for each $0<M<\infty$,

$$
\begin{aligned}
\lim _{\lambda \rightarrow \infty} \lambda^{-2^{k} /\left(2^{k}-1\right)} \ln \left(\mathbf{P}\left\{\left|B^{(k)}(M)\right| \geqslant \lambda\right\}\right) & =\frac{-\left(2^{k+1}-2\right)}{2^{k 2^{k} /\left(2^{k}-1\right)} M^{1 /\left(2^{k}-1\right)}}, \\
\lim _{\lambda \rightarrow \infty} \lambda^{-2^{k} /\left(2^{k}-1\right)} \ln \left(\mathbf{P}\left\{\sup _{0 \leqslant t \leqslant M}\left|B^{(k)}(t)\right| \geqslant \lambda\right\}\right) & =\frac{-\left(2^{k+1}+2\right)}{2^{k 2^{k} /\left(2^{k}-1\right)} M^{1 /\left(2^{k}-1\right)}},
\end{aligned}
$$

where $B^{(k)}=B \stackrel{(k)}{\circ} \circ B(t)$. Our results also apply to compositions of independent Brownian motions. We also consider the iterated fractional Brownian motion. Compact laws of the iterated logarithm for the iterated fractional Brownian motion are obtained.

In Section 6, we give necessary and sufficient conditions for the LDP's for a (nonhomogeneous) Poisson process, under different normalizations. Under some normalization, the LDP does not hold in $l_{\infty}[0, M]$ and we have to consider the LDP in a space of measures.

Given a metric space $(S, d), B(z, \delta)$ denotes the open ball with center $z$ and radius $\delta$. Given a subset $A$ of $S$ and $\delta>0, A(\delta)=\{x \in S: d(x, A)<\delta\}$. In $\mathbf{R}^{d}$, we denote $|z|=\left(\sum_{i=1}^{d} z_{i}^{2}\right)^{1 / 2}$ and $|z|_{\infty}=\max _{1 \leqslant i \leqslant d}\left|z_{i}\right|$, where $z=$ $\left(z_{1}, \ldots, z_{d}\right)$.

2. An extension of the contraction principle. A useful technique to study large deviations is the contraction principle (Donsker and Varadhan, [14, Theorem 2.1]). We will need the following extension of this theorem.

Theorem 2.1. Let $\left\{X_{n}\right\}_{n=1}^{\infty}$ be a sequence of random elements with values in a metric space $\left(S_{1}, d_{1}\right)$. Let $\left\{\varepsilon_{n}\right\}$ be a sequence of positive numbers which converges to zero. Let $\left\{f_{n}\right\}$ be a sequence of Borel functions from $\left(S_{1}, d_{1}\right)$ into $\left(S_{2}, d_{2}\right)$, where $\left(S_{2}, d_{2}\right)$ is a metric space. Let $f$ be a Borel function from $S_{1}$ into $S_{2}$. Suppose that:

(i) $\left\{X_{n}\right\}_{n=1}^{\infty}$ satisfies the LDP with rate $\varepsilon_{n}^{-1}$ and rate function $I_{1}$;

(ii) if $\left\{x_{n}\right\}$ is a sequence in $S_{1}$ such that $x_{n} \rightarrow x$, for some $x$ with $I_{1}(x)<\infty$, then $f_{n}\left(x_{n}\right) \rightarrow f(x)$.

Then

(a) For each open set $U$ in $S_{2}$,

$$
\liminf _{n \rightarrow \infty} \varepsilon_{n} \ln \left(\mathbf{P}_{*}\left\{f_{n}\left(X_{n}\right) \in U\right\}\right) \geqslant-I_{2}(U),
$$

where $I_{2}(y)=\inf \left\{I_{1}(x): f(x)=y\right\}$;

(b) for each closed set $F$ of $S_{2}$,

$$
\limsup _{n \rightarrow \infty} \varepsilon_{n} \ln \left(\mathbf{P}^{*}\left\{f_{n}\left(X_{n}\right) \in F\right\}\right) \leqslant-I_{2}(F) ;
$$

(c) $I_{2}$ is a good rate function. 
P r o o f. To prove part (a), it suffices to show that given $\varepsilon>0$, and $x_{0} \in S_{1}$ with $I_{1}\left(x_{0}\right)<\infty$,

$$
\liminf _{n \rightarrow \infty} \varepsilon_{n} \ln \left(\mathbf{P}_{*}\left\{f_{n}\left(X_{n}\right) \in B\left(y_{0}, \varepsilon\right)\right\}\right) \geqslant-I_{1}\left(x_{0}\right),
$$

where $y_{0}=f\left(x_{0}\right)$. By condition (ii), there are $\delta>0$ and an integer $n_{0}$ such that if $n \geqslant n_{0}$, then $f_{n}\left(B\left(x_{0}, \delta\right)\right) \subset B\left(y_{0}, \varepsilon\right)$. Hence,

$$
\begin{aligned}
& \liminf _{n \rightarrow \infty} \varepsilon_{n} \ln \left(\mathbf{P}_{*}\left\{f_{n}\left(X_{n}\right) \in B\left(y_{0}, \varepsilon\right)\right\}\right) \\
& \quad \geqslant \liminf _{n \rightarrow \infty} \varepsilon_{n} \ln \left(\mathbf{P}_{*}\left\{X_{n} \in B\left(x_{0}, \delta\right)\right\}\right) \geqslant-I_{1}\left(x_{0}\right) .
\end{aligned}
$$

To prove part (b), it suffices to show that given a closed set $F \in S_{2}$,

$$
\limsup _{n \rightarrow \infty} \varepsilon_{n} \ln \left(\mathbf{P}^{*}\left\{f_{n}\left(X_{n}\right) \in F\right\}\right) \leqslant-I_{1}\left(f^{-1}(F)\right) .
$$

For each $\delta>0$ and each positive integer $k$, we have that

$$
\begin{aligned}
& \limsup _{n \rightarrow \infty} \varepsilon_{n} \ln \left(\mathbf{P}^{*}\left\{f_{n}\left(X_{n}\right) \in F\right\}\right) \\
& \quad \leqslant \limsup _{n \rightarrow \infty} \varepsilon_{n} \ln \left(\mathbf{P}^{*}\left\{X_{n} \in G_{k}(\delta)\right\}\right) \leqslant-I_{1}\left(G_{k}(\delta)\right),
\end{aligned}
$$

where $G_{k}(\delta)=\overline{\bigcup_{j=k}^{\infty}\left\{x \in S_{1}: d\left(f_{j}(x), F\right) \leqslant \delta\right\}}$. We need to prove that

$$
I_{1}\left(f^{-1}(F)\right)=\lim _{\delta \rightarrow 0} \lim _{k \rightarrow \infty} I_{1}\left(G_{k}(\delta)\right) \text {. }
$$

Given $x$ with $I_{1}(x)<\infty$ and $f(x) \in F, f_{k}(x) \rightarrow f(x)$. So, for $k$ large enough, $x \in G_{k}(\delta)$. Hence,

$$
I_{1}\left(f^{-1}(F)\right) \geqslant \lim _{\delta \rightarrow 0} \lim _{k \rightarrow \infty} I_{1}\left(G_{k}(\delta)\right)
$$

We may assume that $\lim _{\delta \rightarrow 0} \lim _{k \rightarrow \infty} I_{1}\left(G_{k}(\delta)\right)<\infty$. Take $\delta_{j} \rightarrow 0, k_{j} \nearrow \infty$ and $x_{j} \in G_{k_{j}}\left(\delta_{j}\right)$ such that

$$
\lim _{j \rightarrow \infty} I_{1}\left(x_{j}\right)=\lim _{\delta \rightarrow 0} \lim _{k \rightarrow \infty} I_{1}\left(G_{k}(\delta)\right)
$$

Take $l_{j} \geqslant k_{j}$ and $z_{j}$ with $d\left(f_{l_{j}}\left(z_{j}\right), F\right) \leqslant \delta_{j}$ and $d\left(z_{j}, x_{j}\right) \leqslant j^{-1}$. Since $I_{1}$ is a good rate, there exist a subsequence $\left\{x_{j_{k}}\right\}$ and $x \in S_{1}$ such that $x_{j_{k}} \rightarrow x$. So, $z_{j_{k}} \rightarrow x$ and $f_{l_{j_{k}}}\left(z_{j_{k}}\right) \rightarrow f(x) \in F$. But, by the lower semicontinuity of the function $I_{1}, I_{1}(x) \leqslant \liminf _{j \rightarrow \infty} I_{1}\left(x_{j}\right)$. Therefore, $(2.1)$ follows.

To prove part (c), it suffices to show that the restriction of $f$ to $\left\{x: I_{1}(x)<\infty\right\}$ is continuous. Note that for each $c \geqslant 0,\left\{y: I_{2}(y) \leqslant c\right\}=$ $f\left(\left\{x: I_{1}(x) \leqslant c\right\}\right)$. Given $\varepsilon>0$ and $x_{0} \in\left\{x: I_{1}(x)<\infty\right\}$, there are $\delta>0$ and a positive integer $n_{0}$ such that for each $n \geqslant n_{0}, f_{n}\left(B\left(x_{0}, \delta\right)\right) \subset B\left(f\left(x_{0}\right), \varepsilon\right)$. Given $x \in B\left(x_{0}, \delta\right) \cap\left\{x: I_{1}(x)<\infty\right\}$, for $n \geqslant n_{0}, d_{2}\left(f_{n}(x), f\left(x_{0}\right)\right)<\varepsilon$. From this and hypothesis (ii), for each $x \in B\left(x_{0}, \delta\right) \cap\left\{x: I_{1}(x)<\infty\right\}$, we have $d_{2}\left(f(x), f\left(x_{0}\right)\right)<\varepsilon$. Theorem 2.1 is proved.

The next corollary follows immediately from the previous theorem. 
Corollary 2.1. Let $\left\{X_{n}\right\}_{n=1}^{\infty}$ be a sequence of random elements with values in a metric space $\left(S_{1}, d_{1}\right)$ such that $\left\{X_{n}\right\}_{n=1}^{\infty}$ satisfies the LDP with rate $\varepsilon_{n}^{-1}$ and rate function $I_{1}$. Let $f:\left(S_{1}, d_{1}\right) \rightarrow\left(S_{2}, d_{2}\right)$ be a function which is continuous at each $x$ with $I_{1}(x)<\infty$, where $\left(S_{2}, d_{2}\right)$ is a metric space. Then $\left\{f\left(X_{n}\right)\right\}_{n=1}^{\infty}$ satisfies the LDP with rate $\varepsilon_{n}^{-1}$ and rate function $I_{2}(y)=$ $\inf \left\{I_{1}(x): f(x)=y\right\}$.

\section{Asymptotic equicontinuity for the large deviation principle.}

In this section, we prove that the LDP in $l_{\infty}(T)$ is equivalent to the LDP for the finite dimensional distributions plus an exponential asymptotic equicontinuity condition with respect to certain pseudometric, which makes $T$ totally bounded. This condition can be interpreted as a tightness condition. Assuming the LDP for the finite dimensional distributions, we claim that for each $k \geqslant 1$,

$$
\rho_{k}(s, t)=\sup \left\{\left|u_{2}-u_{1}\right|: I_{s, t}\left(u_{1}, u_{2}\right) \leqslant k\right\}
$$

defines a pseudometric on $T$. The LDP for the finite dimensional distributions implies that for each $s, t \in T$ and each $k \geqslant 1,\left\{\left(u_{1}, u_{2}\right): I_{s, t}\left(u_{1}, u_{2}\right) \leqslant k\right\}$ is a compact set. So, for each $s, t \in T, \rho_{k}(s, t)<\infty$. By the contraction principle, given $r, s, t \in T$,

$$
\begin{aligned}
\rho_{k}(r, t)= & \sup \left\{\left|u_{2}-u_{1}\right|: I_{r, t}\left(u_{1}, u_{2}\right) \leqslant k\right\} \\
= & \sup \left\{\left|u_{3}-u_{1}\right|: I_{r, s, t}\left(u_{1}, u_{2}, u_{3}\right) \leqslant k\right\} \\
\leqslant & \sup \left\{\left|u_{2}-u_{1}\right|: I_{r, s, t}\left(u_{1}, u_{2}, u_{3}\right) \leqslant k\right\} \\
& +\sup \left\{\left|u_{3}-u_{2}\right|: I_{r, s, t}\left(u_{1}, u_{2}, u_{3}\right) \leqslant k\right\}=\rho_{k}(r, s)+\rho_{k}(s, t) .
\end{aligned}
$$

Therefore, $\rho_{k}$ is a pseudometric. The pseudometrics $\rho_{k}$ play a role in the exponential asymptotic tightness of a sequence of stochastic processes.

First, we prove the following lemma.

Lemma 3.1. Let $\left\{U_{n}(t): t \in T\right\}$ be a sequence of stochastic processes, where $T$ is an index set. Let $\left\{\varepsilon_{n}\right\}$ be a sequence of positive numbers that converges to zero. Suppose that:

(i) For each $t_{1}, \ldots, t_{m} \in T,\left(U_{n}\left(t_{1}\right), \ldots, U_{n}\left(t_{m}\right)\right)$ satisfies the LDP with speed $\varepsilon_{n}^{-1}$ and good rate function $I_{t_{1}, \ldots, t_{m}}$;

(ii) for each $k \geqslant 1,\left(T, \rho_{k}\right)$ is a totally bounded pseudometric space.

Then, for each $0 \leqslant c<\infty,\left\{z \in l_{\infty}(T): I(z) \leqslant c\right\}$ is a set of uniformly bounded and uniformly equicontinuous functions in $(T, \rho)$ and it is closed in $l_{\infty}(T)$, where

$$
I(z)=\sup \left\{I_{t_{1}, \ldots, t_{m}}\left(z\left(t_{1}\right), \ldots, z\left(t_{m}\right)\right): t_{1}, \ldots, t_{m} \in T, m \geqslant 1\right\}
$$

and

$$
\rho(s, t)=\sum_{k=1}^{\infty} k^{-2} \min \left(\rho_{k}(s, t), 1\right) .
$$


Consequently, for each $0 \leqslant c<\infty,\left\{z \in l_{\infty}(T): I(z) \leqslant c\right\}$ is a compact set of $l_{\infty}(T)$.

P r o o f. Since each $I_{t_{1}, \ldots, t_{m}}$ is lower semicontinuous, so is $I(\cdot)$. This implies that for each $0 \leqslant c<\infty$ the set $\left\{z \in l_{\infty}(T): I(z) \leqslant c\right\}$ is closed. Let $\rho_{k}^{*}(s, t)=\sup \{|z(t)-z(s)|: I(z) \leqslant k\}$ and let $\rho^{*}(s, t)=$ $\sum_{k=1}^{\infty} k^{-2} \min \left(\rho_{k}^{*}(s, t), 1\right)$. It is easy to see that the set of functions $\left\{z \in l_{\infty}(T): I(z) \leqslant k\right\}$ is a set of uniformly equicontinuous functions in $\left(T, \rho^{*}\right)$. Since $I_{s, t}(z(s), z(t)) \leqslant I(z)$, we have $\rho_{k}^{*}(s, t) \leqslant \rho_{k}(s, t)$. Hence, the set of functions $\left\{z \in l_{\infty}(T): I(z) \leqslant k\right\}$ is a set of uniformly equicontinuous functions in $(T, \rho)$. Since for each $t \in T, I_{t}$ is a good rate function, for each $0 \leqslant c<\infty$,

$$
\sup \{|z(t)|: I(z) \leqslant c\} \leqslant \sup \left\{|u|: I_{t}(u) \leqslant c\right\}<\infty .
$$

So, $\left\{z \in l_{\infty}(T): I(z) \leqslant c\right\}$ is a set of uniformly bounded functions. The Arzelà-Ascoli theorem (see for example Theorem IV.6.7 in [16]) implies that $\left\{z \in l_{\infty}(T): I(z) \leqslant c\right\}$ is a compact set of $l_{\infty}(T)$. We may apply this theorem even when $(T, \rho)$ is a totally bounded pseudometric space and not a compact space because identifying the points which are at a zero distance (see Problem $2 \mathrm{C}$ in [34]), we may assume that $(T, \rho)$ is a metric space and imbedding $T$ in its completion, we may assume that $(T, \rho)$ is complete. A metric space can be isometrically embedded as a dense subset of the complete metric space consisting of the Cauchy sequences in this space (see for example Theorem 24.4 in [34]). The considered functions can be extended as functions in the completion by the principle of extension by continuity (see Theorem I.6.17 in [16]). Lemma 3.1 is proved.

We call a function $\pi: T \rightarrow T$ a finite partition function if for each $t \in T, \pi(\pi(t))=\pi(t)$, and the cardinality of $\{\pi(t): t \in T\}$ is finite. Let $\pi(T)=\left\{t_{1}, \ldots, t_{m}\right\}$ and $A_{j}=\left\{t \in T: \pi(t)=t_{j}\right\}$ for $1 \leqslant j \leqslant m$, then $\left\{A_{1}, \ldots, A_{m}\right\}$ is a partition of $T$. Finite partition functions can be used to characterize compactness of $l_{\infty}(T)$. A set $K$ of $l_{\infty}(T)$ is compact if and only if it is closed, bounded and for each $\tau>0$, there exists a finite partition function $\pi: T \rightarrow T$ such that $\sup _{x \in K}|x(t)-x(\pi(t))| \leqslant \tau$ (see Theorem IV.5.6 in [16]). We also have that if $K$ is a compact set of $l_{\infty}(T)$, then $K$ is a set of uniformly bounded and equicontinuous functions in the pseudometric space $(T, d)$, where $d(s, t)=\sup _{x \in K}|x(s)-x(t)|$.

Theorem 3.1. Let $\left\{U_{n}(t): t \in T\right\}$ be a sequence of stochastic processes, where $T$ is an index set. Let $\left\{\varepsilon_{n}\right\}$ be a sequence of positive numbers that converges to zero. Let $I: l_{\infty}(T) \rightarrow[0, \infty]$ and let $I_{t_{1}, \ldots, t_{m}}: \mathbf{R}^{m} \rightarrow[0, \infty]$ be a function for each $t_{1}, \ldots, t_{m} \in T$. Let d be a pseudometric in $T$.

Consider the following conditions.

(a.1) $(T, d)$ is totally bounded. 
(a.2) For each $t_{1}, \ldots, t_{m} \in T,\left(U_{n}\left(t_{1}\right), \ldots, U_{n}\left(t_{m}\right)\right)$ satisfies the LDP with speed $\varepsilon_{n}^{-1}$ and good rate function $I_{t_{1}, \ldots, t_{m}}$.

(a.3) For each $\tau>0$,

$$
\lim _{\eta \rightarrow 0} \limsup _{n \rightarrow \infty} \varepsilon_{n} \ln \left(\mathbf{P}^{*}\left\{\sup _{d(s, t) \leqslant \eta}\left|U_{n}(t)-U_{n}(s)\right| \geqslant \tau\right\}\right)=-\infty .
$$

(b.1) For each $0 \leqslant c<\infty,\left\{z \in l_{\infty}(T): I(z) \leqslant c\right\}$ is a compact set of $l_{\infty}(T)$.

(b.2) For each $A \subset l_{\infty}(T)$,

$$
\begin{aligned}
-\inf _{z \in A^{\circ}} I(z) & \leqslant \liminf _{n \rightarrow \infty} \varepsilon_{n} \ln \left(\mathbf{P}_{*}\left\{U_{n} \in A\right\}\right) \\
& \leqslant \limsup _{n \rightarrow \infty} \varepsilon_{n} \ln \left(\mathbf{P}^{*}\left\{U_{n} \in A\right\}\right) \leqslant-\inf _{z \in \bar{A}} I(z) .
\end{aligned}
$$

If the set of conditions (a) is satisfied, then the set of conditions (b) holds with $I(\cdot)$ given by (3.2).

If the set of conditions (b) is satisfied, then the set of conditions (a) holds with

$$
\begin{aligned}
& I_{t_{1}, \ldots, t_{m}}\left(u_{1}, \ldots, u_{m}\right) \\
& \quad=\inf \left\{I(z): z \in l_{\infty}(T),\left(z\left(t_{1}\right), \ldots, z\left(t_{m}\right)\right)=\left(u_{1}, \ldots, u_{m}\right)\right\}
\end{aligned}
$$

and the pseudometric $\rho$ in (3.3).

P r o o f. Assume that the set of conditions (a) holds. First, we show that for each $k \geqslant 1$,

$$
\lim _{\eta \rightarrow 0} \sup _{d(s, t) \leqslant \eta} \rho_{k}(s, t)=0 .
$$

Given $\tau>0$, take $\eta>0$, such that

$$
\limsup _{n \rightarrow \infty} \varepsilon_{n} \ln \left(\mathbf{P}^{*}\left\{\sup _{d(s, t) \leqslant \eta}\left|U_{n}(t)-U_{n}(s)\right| \geqslant \tau\right\}\right) \leqslant-k-1 .
$$

This implies that

$$
\sup _{d(s, t) \leqslant \eta} \limsup _{n \rightarrow \infty} \varepsilon_{n} \ln \left(\mathbf{P}\left\{\left|U_{n}(t)-U_{n}(s)\right| \geqslant \tau\right\}\right) \leqslant-k-1,
$$

which gives that $\sup _{d(s, t) \leqslant \eta} \rho_{k}(s, t) \leqslant \tau$. Therefore, (3.5) holds. This implies that for each $k \geqslant 1,\left(T, \rho_{k}\right)$ is totally bounded. Hence (b.1) follows from Lemma 3.1.

Define

$$
\begin{aligned}
& I_{t_{1}, \ldots, t_{m}}^{(1)}\left(u_{1}, \ldots, u_{m}\right) \\
& \quad=\inf \left\{I(z): z \in l_{\infty}(T),\left(z\left(t_{1}\right), \ldots, z\left(t_{m}\right)\right)=\left(u_{1}, \ldots, u_{m}\right)\right\}
\end{aligned}
$$


where $I(\cdot)$ is defined in (3.2). We claim that for each $t_{1}, \ldots, t_{m} \in T$ and each $u_{1}, \ldots, u_{m} \in \mathbf{R}$,

$$
I_{t_{1}, \ldots, t_{m}}\left(u_{1}, \ldots, u_{m}\right)=I_{t_{1}, \ldots, t_{m}}^{(1)}\left(u_{1}, \ldots, u_{m}\right) .
$$

It is easy to see that $I_{t_{1}, \ldots, t_{m}}\left(u_{1}, \ldots, u_{m}\right) \leqslant I_{t_{1}, \ldots, t_{m}}^{(1)}\left(u_{1}, \ldots, u_{m}\right)$. To prove the inverse inequality, we may assume that $I_{t_{1}, \ldots, t_{m}}\left(u_{1}, \ldots, u_{m}\right)<\infty$. Let $\tau>0$ and let $k_{0}>2 \tau+I_{t_{1}, \ldots, t_{m}}\left(u_{1}, \ldots, u_{m}\right)$. We can find $t_{m+1}, t_{m+2}, \ldots$ such that $\left\{t_{n}\right\}_{n=1}^{\infty}$ is a dense set in $\left(T, \rho_{k_{0}}\right)$. By the contraction principle, for each $r_{1}, \ldots, r_{m}, s_{1}, \ldots, s_{p} \in T$ and each $u_{1}, \ldots, u_{m} \in \mathbf{R}$,

$$
\begin{aligned}
& I_{r_{1}, \ldots, r_{m}}\left(u_{1}, \ldots, u_{m}\right) \\
& \quad=\inf \left\{I_{r_{1}, \ldots, r_{m}, s_{1}, \ldots, s_{p}}\left(u_{1}, \ldots, u_{m}, v_{1}, \ldots, v_{p}\right): v_{1}, \ldots, v_{p} \in \mathbf{R}\right\} .
\end{aligned}
$$

So, we can find $u_{m+1}, u_{m+2}, \ldots$ such that for each $n \geqslant m$,

$$
I_{t_{1}, \ldots, t_{n}}\left(u_{1}, \ldots, u_{n}\right)<\tau+I_{t_{1}, \ldots, t_{m}}\left(u_{1}, \ldots, u_{m}\right) \text {. }
$$

Define $z\left(t_{j}\right)=u_{j}$. By the definition of the pseudometric $\rho_{k_{0}}$, we have that $z$ is an equicontinuous function in $\left(\left\{t_{n}\right\}_{n=1}^{\infty}, \rho_{k_{0}}\right)$. So, there exists a unique extension of $z$ to an equicontinuous function in $\left(T, \rho_{k_{0}}\right)$ (see Theorem I.6.17 in [16]). By an abuse of notation, we call this extension $z$. To finish the proof of (3.6), it suffices to show that

$$
I(z) \leqslant 2 \tau+I_{t_{1}, \ldots, t_{m}}\left(u_{1}, \ldots, u_{m}\right) .
$$

Hence, we need to prove that for each $s_{1}, \ldots, s_{l} \in T$,

$$
I_{s_{1}, \ldots, s_{l}}\left(z\left(s_{1}\right), \ldots, z\left(s_{l}\right)\right) \leqslant \tau+I_{t_{1}, \ldots, t_{m}}\left(u_{1}, \ldots, u_{m}\right)
$$

For each $1 \leqslant i \leqslant l$, take $t_{n_{j}^{(i)}}$ such that $\rho_{k_{0}}\left(t_{n_{j}^{(i)}}, s_{i}\right) \rightarrow 0$, as $j \rightarrow \infty$. By (3.7), there are $v_{i}^{(j)}$ such that

$$
\begin{aligned}
& I_{t_{n_{j}^{(1)}, \ldots, t_{n_{j}^{(m)}}, s_{1}, \ldots, s_{l}}}\left(z\left(t_{n_{j}^{(1)}}\right), \ldots, z\left(t_{n_{j}^{(m)}}\right), v_{1}^{(j)}, \ldots, v_{l}^{(j)}\right) \\
& \quad \leqslant I_{t_{n_{j}^{(1)}, \ldots, t_{n_{j}^{(m)}}}}\left(z\left(t_{n_{j}^{(1)}}\right), \ldots, z\left(t_{n_{j}^{(m)}}\right)\right)+\tau \\
& \quad<I_{t_{1}, \ldots, t_{m}}\left(u_{1}, \ldots, u_{m}\right)+2 \tau .
\end{aligned}
$$

Hence, $I_{t_{n_{j}^{(i)}}, s_{i}}\left(z\left(t_{n_{j}^{(i)}}\right), v_{i}^{(j)}\right) \leqslant k_{0}$. So, $z\left(t_{n_{j}^{(i)}}\right)-v_{i}^{(j)} \rightarrow 0$, as $j \rightarrow \infty$. Hence, $v_{i}^{(j)} \rightarrow z\left(s_{i}\right)$, as $j \rightarrow \infty$. From (3.7) and (3.10), $I_{s_{1}, \ldots, s_{l}}\left(v_{1}^{(j)}, \ldots, v_{l}^{(j)}\right)<$ $2 \tau+I_{t_{1}, \ldots, t_{m}}\left(u_{1}, \ldots, u_{m}\right)$. This inequality and the lower semicontinuity of $I_{s_{1}, \ldots, s_{l}}$ imply $(3.9)$.

In order to prove that for each set $A \subset l_{\infty}(T)$,

$$
\limsup _{n \rightarrow \infty} \varepsilon_{n} \ln \left(\mathbf{P}^{*}\left\{U_{n} \in A\right\}\right) \leqslant-\inf _{z \in \bar{A}} I(z),
$$


we may suppose that $\inf _{z \in \bar{A}} I(z)>0$. Let $0<a<\inf _{z \in \bar{A}} I(z)$ and let $K=\left\{z \in l_{\infty}(T): I(z) \leqslant a\right\}$. Then $K \cap \bar{A}=\varnothing$ and $K$ is a compact set. Thus, there exists a $\delta>0$ such that $K(4 \delta) \cap \bar{A}=\varnothing$. By Lemma 3.1, (3.5) and (a.3), there exists an $\eta>0$ such that

$$
\begin{gathered}
\sup _{z \in K} \sup _{d(s, t) \leqslant \eta}|z(s)-z(t)| \leqslant \delta, \\
\limsup _{n \rightarrow \infty} \varepsilon_{n} \ln \left(\mathbf{P}^{*}\left\{\sup _{d(s, t) \leqslant \eta}\left|U_{n}(t)-U_{n}(s)\right| \geqslant \delta\right\}\right) \leqslant-2 a .
\end{gathered}
$$

By condition (a.1) there exists a finite partition function $\pi: T \rightarrow T$ such that $\sup _{t \in T} d(t, \pi(t)) \leqslant \eta$. Let $\pi(T)=\left\{t_{1}, \ldots, t_{m}\right\}$ and let $C_{\delta}=$ $\left\{\left(z\left(t_{1}\right), \ldots, z\left(t_{m}\right)\right): z \in K(\delta)\right\}$. It is easy to see that if $\left(z\left(t_{1}\right), \ldots, z\left(t_{m}\right)\right) \in C_{\delta}$ and $\sup _{t \in T}|z(t)-z(\pi(t))| \leqslant \delta$, then $z \in K(4 \delta)$. Hence

$$
\begin{aligned}
\mathbf{P}^{*}\left\{U_{n} \in \bar{A}\right\} \leqslant & \mathbf{P}^{*}\left\{U_{n} \notin K(4 \delta)\right\} \leqslant \mathbf{P}^{*}\left\{\left(U_{n}\left(t_{1}\right), \ldots, U_{n}\left(t_{m}\right)\right) \notin C_{\delta}\right\} \\
& +\mathbf{P}^{*}\left\{\sup _{t \in T}\left|U_{n}(t)-U_{n}(\pi(t))\right| \geqslant \delta\right\} .
\end{aligned}
$$

Since $C_{\delta}$ is an open set, by the LDP for the finite dimensional distributions,

$$
\begin{aligned}
& \limsup _{n \rightarrow \infty} \varepsilon_{n} \ln \left(\mathbf{P}^{*}\left\{\left(U_{n}\left(t_{1}\right), \ldots, U_{n}\left(t_{m}\right)\right) \notin C_{\delta}\right\}\right) \\
& \quad \leqslant-\inf \left\{I_{t_{1}, \ldots, t_{m}}\left(u_{1}, \ldots, u_{m}\right):\left(u_{1}, \ldots, u_{m}\right) \notin C_{\delta}\right\} .
\end{aligned}
$$

By (3.6),

$$
\inf \left\{I_{t_{1}, \ldots, t_{m}}\left(u_{1}, \ldots, u_{m}\right):\left(u_{1}, \ldots, u_{m}\right) \notin C_{\delta}\right\} \geqslant \inf \{I(z): z \notin K(\delta)\} \geqslant a .
$$

So, $\lim \sup _{n \rightarrow \infty} \varepsilon_{n} \ln \left(\mathbf{P}^{*}\left\{U_{n} \in A\right\}\right) \leqslant-a$. Letting $a \rightarrow \inf _{z \in \bar{A}} I(z)$, (3.11) follows.

Next, we prove that for each set $A \subset l_{\infty}(T)$,

$$
-\inf _{z \in A^{\circ}} I(z) \leqslant \liminf _{n \rightarrow \infty} \varepsilon_{n} \ln \left(\mathbf{P}_{*}\left\{U_{n} \in A\right\}\right) .
$$

It suffices to prove that if $z_{0} \in A^{\circ}$ and $I\left(z_{0}\right)<\infty$, then

$$
-I\left(z_{0}\right) \leqslant \liminf _{n \rightarrow \infty} \varepsilon_{n} \ln \left(\mathbf{P}_{*}\left\{U_{n} \in A\right\}\right) .
$$

Take $a>I\left(z_{0}\right)>b$. Let $K=\left\{z \in l_{\infty}(T): I(z) \leqslant a\right\}$. There exists a $\delta>0$ such that $B\left(z_{0}, 3 \delta\right) \subset A^{\circ}$ and $\inf \left\{I(z): z \in B\left(z_{0}, 3 \delta\right)\right\}>b$. By Lemma 3.1, (3.5) and (a.3) there exists an $\eta>0$ such that (3.12) and (3.13) hold. Take a finite partition function $\pi$ such that $\sup _{t \in T} d(t, \pi(t)) \leqslant \eta$. 
Let $\left\{t_{1}, \ldots, t_{m}\right\}=\pi(T)$. Then, if $\max _{1 \leqslant j \leqslant m}\left|z\left(t_{j}\right)-z_{0}\left(t_{j}\right)\right|<\delta$ and $\sup _{d(s, t) \leqslant \eta}|z(s)-z(t)|<\delta$, we have $\sup _{t \in T}\left|z(t)-z_{0}(t)\right|<3 \delta$. So,

$$
\begin{aligned}
\mathbf{P}\left\{\max _{1 \leqslant j \leqslant m}\left|U_{n}\left(t_{j}\right)-z_{0}\left(t_{j}\right)\right|<\delta\right\} \leqslant & \mathbf{P}_{*}\left\{\sup _{t \in T}\left|U_{n}(t)-z_{0}(t)\right|<3 \delta\right\} \\
& +\mathbf{P}^{*}\left\{\sup _{d(s, t) \leqslant \eta}\left|U_{n}(s)-U_{n}(t)\right| \geqslant \delta\right\} .
\end{aligned}
$$

We claim that

$$
\left\{z \in l_{\infty}(T): \sup _{1 \leqslant j \leqslant m}\left|z\left(t_{j}\right)-z_{0}\left(t_{j}\right)\right|<\delta\right\} \subset\left\{z \in l_{\infty}(T): I(z) \geqslant b\right\} .
$$

If $\sup _{1 \leqslant j \leqslant m}\left|z\left(t_{j}\right)-z_{0}\left(t_{j}\right)\right|<\delta$ and $z \notin K$, then $I(z)>a>b$. If $\sup _{1 \leqslant j \leqslant m}\left|z\left(t_{j}\right)-z_{0}\left(t_{j}\right)\right|<\delta$ and $z \in K$, then $z \in B\left(z_{0}, 3 \delta\right)$ and $I(z)>b$. Therefore; (3.15) holds. So, by condition (a.2),

$$
-b \leqslant \liminf _{n \rightarrow \infty} \varepsilon_{n} \ln \left(\mathbf{P}\left\{\max _{1 \leqslant j \leqslant m}\left|U_{n}\left(t_{j}\right)-z_{0}\left(t_{j}\right)\right|<\delta\right\}\right) .
$$

Therefore, (3.14) follows.

Assume that the set of conditions (b) holds. It is easy to see that if $K$ is a compact set of $l_{\infty}(T)$ and $e(s, t)=\sup _{z \in K}|z(s)-z(t)|$, then $(T, e)$ is a totally bounded pseudometric space and $K$ is a collection of uniformly bounded and uniformly e-equicontinuous functions. Hence, for each $k \geqslant 1$, $\left(T, \rho_{k}^{*}\right)$ is a totally bounded pseudometric space, where $\rho_{k}^{*}$ is as in the proof of Lemma 3.1. The equality (3.6) implies that $\rho_{k}^{*}=\rho_{k}$ ((3.6) follows from the contraction principle). Therefore, $(T, \rho)$ is also totally bounded, that is, (a.1) holds.

Given $t_{1}, \ldots, t_{m} \in T$, the function $g: l_{\infty}(T) \rightarrow \mathbf{R}^{m}$ defined by $g(z)=$ $\left(z\left(t_{1}\right), \ldots, z\left(t_{m}\right)\right)$ is a continuous function. So, the contraction principle implies (a.2).

To prove (a.3), it suffices to show that given $0<\tau, c<\infty$, there exists an $\eta>0$ such that

$$
\limsup _{n \rightarrow \infty} \varepsilon_{n} \ln \mathbf{P}^{*}\left\{\sup _{d(s, t) \leqslant \eta}\left|U_{n}(t)-U_{n}(s)\right| \geqslant \tau\right\} \leqslant-c .
$$

The set $\left\{z \in l_{\infty}(T): I(z) \leqslant c\right\}$ is a set of uniformly bounded and uniformly $\rho$-continuous functions. So, there exists an $\eta>0$ such that

$$
\left\{z \in l_{\infty}(T): I(z) \leqslant c\right\} \subset\left\{z \in l_{\infty}(T): \sup _{\rho(s, t) \leqslant \eta}|z(s)-z(t)| \leqslant 2^{-1} \tau\right\} .
$$

So, $F:=\left\{z \in l_{\infty}(T): \sup _{\rho(s, t) \leqslant \eta}|z(s)-z(t)| \geqslant \tau\right\}$ is closed in $l_{\infty}(T)$ and $\inf _{z \in F} I(z) \geqslant c$. Hence, (3.16) holds. Theorem 3.1 is proved. 
Of course, if conditions (a) in Theorem 3.1 hold for some pseudometric $d$ and $e$ is uniformly equivalent to $d$, then conditions (a) hold for $e$. Pseudometrics $d$ and $e$ are uniformly equivalent if

$$
\lim _{\delta \rightarrow 0} \sup _{d(s, t) \leqslant \delta} e(s, t)=\lim _{\delta \rightarrow 0} \sup _{e(s, t) \leqslant \delta} d(s, t)=0 .
$$

Theorem 3.1 is true with $\rho^{*}$ instead of $\rho$.

Alternatively, conditions (a.1) and (a.3) in Theorem 3.1 can be put using finite partition functions. Conditions (a.1) and (a.3) are equivalent to the following one: for each $c, \eta>0$, there exists a finite partition function $\pi$ of $T$ such that

$$
\limsup _{n \rightarrow \infty} \varepsilon_{n} \ln \mathbf{P}^{*}\left\{\sup _{t \in T}\left|U_{n}(t)-U_{n}(\pi(t))\right| \geqslant \eta\right\} \leqslant-c .
$$

Under the conditions in Theorem 3.1, if $z \in l_{\infty}(T)$ and $I(z)<\infty$, then $z$ is uniformly continuous in $(T, d)$.

The next corollary characterizes when the asymptotic equicontinuity condition is satisfied with respect to the Euclidean distance if $T$ is a bounded set of $\mathbf{R}^{d}$.

Corollary 3.1. Let $T$ be a compact set of $\mathbf{R}^{d}$, let $\left\{U_{n}(t): t \in T\right\}$ be a sequence of stochastic processes and let $\left\{\varepsilon_{n}\right\}$ be a sequence of positive numbers that converges to zero. Then the following sets of conditions ((a) and (b)) are equivalent.

(a.1) $\left\{U_{n}(t): t \in T\right\}$ satisfies the $L D P$ in $l_{\infty}(T)$ with speed $\varepsilon_{n}^{-1}$.

(a.2) For each $t_{0} \in T, \lim _{t \rightarrow t_{0}} \rho\left(t, t_{0}\right)=0$.

(b.1) For each $t_{1}, \ldots, t_{m} \in T,\left(U_{n}\left(t_{1}\right), \ldots, U_{n}\left(t_{m}\right)\right)$ satisfies the LDP with speed $\varepsilon_{n}^{-1}$ and good rate function $I_{t_{1}, \ldots, t_{m}}$.

(b.2) For each $\tau>0$,

$$
\lim _{\eta \rightarrow 0} \limsup _{n \rightarrow \infty} \varepsilon_{n} \ln \left(\mathbf{P}^{*}\left\{\sup _{s, t \in T,|s-t| \leqslant \eta}\left|U_{n}(t)-U_{n}(s)\right| \geqslant \tau\right\}\right)=-\infty .
$$

P r o of. Assume the conditions (a). The condition (b.1) follows from (a.1) and the contraction principle. We have that the identity function $(T,|\cdot|) \rightarrow(T, \rho)$ is continuous, where $\rho$ is as in $(3.3)$. Since $(T,|\cdot|)$ is a compact set, this function is also uniformly continuous. So, (b.2) holds.

Assume the set of conditions (b). By Theorem 3.1, (a.1) holds. Given $\tau>0$ and $k>0$, there exists an $\eta>0$ such that

$$
\limsup _{n \rightarrow \infty} \varepsilon_{n} \ln \left(\mathbf{P}^{*}\left\{\sup _{s, t \in T,|s-t| \leqslant \eta}\left|U_{n}(t)-U_{n}(s)\right| \geqslant \tau\right\}\right) \leqslant-k-1 .
$$

Hence, if $|s-t| \leqslant \eta$, then

$$
\limsup _{n \rightarrow \infty} \varepsilon_{n} \ln \left(\mathbf{P}\left\{\left|U_{n}(t)-U_{n}(s)\right| \geqslant \tau\right\}\right) \leqslant-k-1 .
$$


By the LDP for $\left(U_{n}(s), U_{n}(t)\right)$,

$$
\liminf _{n \rightarrow \infty} \varepsilon_{n} \ln \left(\mathbf{P}\left\{\left|U_{n}(t)-U_{n}(s)\right|>\tau\right\}\right) \geqslant-\inf \left\{I_{s, t}\left(u_{1}, u_{2}\right):\left|u_{1}-u_{2}\right|>\tau\right\} .
$$

Therefore, for $|s-t| \leqslant \eta$,

$$
k<\inf \left\{I_{s, t}\left(u_{1}, u_{2}\right):\left|u_{1}-u_{2}\right|>\tau\right\} .
$$

This implies that $\sup _{s, t \in T,|s-t| \leqslant \eta} \sup \left\{\left|u_{2}-u_{1}\right|: I_{s, t}\left(u_{1}, u_{2}\right) \mid \leqslant k\right\} \leqslant \tau$. Corollary 3.1 is proved.

Observe that in the previous corollary, the condition (a.2) can be replaced by the condition:

$(\text { a.2 })^{\prime}$ For each $0<k<\infty$,

$$
\lim _{\eta \rightarrow 0} \sup _{s, t \in T,|s-t| \leqslant \eta} \sup \left\{\left|u_{2}-u_{1}\right|: I_{s, t}\left(u_{1}, u_{2}\right) \mid \leqslant k\right\}=0 .
$$

It may happen that a sequence of stochastic processes $\left\{U_{n}(t)\right.$ : $0 \leqslant t \leqslant M\}$ satisfies the LDP, but condition (b.2) in the previous theorem is not satisfied. For the stochastic processes in Theorem 6.2 (see Part II), if $(\mu[0, M n])^{-1} \mu[0, M n) \rightarrow 0$, the LDP holds, but neither (b.2) nor (a.2) in the previous theorem hold.

The next corollary allows one to combine the LDP for several index sets.

Corollary 3.2. Let $\left\{U_{n}(t): t \in T\right\}$ be a sequence of stochastic processes with values in $\mathbf{R}^{d}$. Let $T^{(1)}$ and $T^{(2)}$ be two subsets of $T$ such that $T=$ $T^{(1)} \cup T^{(2)}$. Suppose that:

(i) For each $t_{1}, \ldots, t_{m} \in T,\left(U_{n}\left(t_{1}\right), \ldots, U_{n}\left(t_{m}\right)\right)$ satisfies the LDP with speed $\varepsilon_{n}^{-1}$ and rate function $I_{t_{1}, \ldots, t_{n}}$;

(ii) for each $j=1,2,\left\{U_{n}(t): t \in T_{j}\right\}$ satisfies the $L D P l_{\infty}\left(T_{j}\right)$ with speed $\varepsilon_{n}^{-1}$.

Then $\left\{U_{n}(t): t \in T\right\}$ satisfies the $L D P$ in $l_{\infty}(T)$ with speed $\varepsilon_{n}^{-1}$ and rate function

$$
I(z)=\sup \left\{I_{t_{1}, \ldots, t_{n}}\left(z\left(t_{1}\right), \ldots, z\left(t_{n}\right)\right): t_{1}, \ldots, t_{m} \in T, m \geqslant 1\right\} .
$$

P r o o f. Given $c, \tau>0$, there exist partition functions $\pi^{(i)}, i=1,2$, such that

$$
\limsup _{n \rightarrow \infty} \varepsilon_{n} \ln \left(\mathbf{P}^{*}\left\{\sup _{t \in T^{(i)}}\left|U_{n}(t)-U_{n}\left(\pi^{(i)}(t)\right)\right| \geqslant \eta\right\}\right) \leqslant-c .
$$

Let $\pi(t)=\pi^{(1)}(t)$, if $t \in T^{(1)}$, and $\pi(t)=\pi^{(2)}(t)$, if $t \in T^{(2)}-T^{(1)}$. Then

$$
\limsup _{n \rightarrow \infty} \varepsilon_{n} \ln \left(\mathbf{P}^{*}\left\{\sup _{t \in T}\left|U_{n}(t)-U_{n}(\pi(t))\right| \geqslant 2 \eta\right\}\right) \leqslant-c
$$

which implies the claim. Corollary 3.2 is proved. in $\mathbf{R}^{d}$.

The next corollary allows one to obtain the LDP for stochastic processes 
Corollary 3.3. Let $\left\{U_{n}^{(i)}(t): t \in T\right\}, 1 \leqslant i \leqslant d$, be sequences of stochastic processes defined on the same probability space. Suppose that:

(i) For each $t_{1}, \ldots, t_{m} \in T$,

$$
\left(U_{n}^{(1)}\left(t_{1}\right), \ldots, U_{n}^{(1)}\left(t_{m}\right), \ldots, U_{n}^{(d)}\left(t_{1}\right), \ldots, U_{n}^{(d)}\left(t_{m}\right)\right)
$$

satisfies the LDP in $\mathbf{R}^{d m}$ with speed $\varepsilon_{n}^{-1}$;

(ii) for each $1 \leqslant i \leqslant d,\left\{U_{n}^{(i)}(t): t \in T\right\}$ satisfies the $L D P$ in $l_{\infty}(T)$ with speed $\varepsilon_{n}^{-1}$.

Then $\left\{\left(U_{n}^{(1)}(t), \ldots, U_{n}^{(d)}(t)\right): t \in T\right\}$ satisfies the LDP in $l_{\infty}\left(T, \mathbf{R}^{d}\right)$ with speed $\varepsilon_{n}^{-1}$.

P r o o f. Let $T^{*}=\{1, \ldots, m\} \times T$. Let $W_{n}(i, t)=U_{n}^{(i)}(t)$ for $t \in T$. By assumption (ii), implies that for each $1 \leqslant i \leqslant d,\left\{W_{n}(i, t): t \in T\right\}$ satisfies the LDP with speed $\varepsilon_{n}^{-1}$. By Corollary 3.2, $\left\{W_{n}\left(t^{*}\right): t^{*} \in T^{*}\right\}$ satisfies the LDP in $l_{\infty}\left(T^{*}\right)$ with speed $\varepsilon_{n}^{-1}$. The space $l_{\infty}\left(T^{*}\right)$ is isometric to $l_{\infty}\left(T, \mathbf{R}^{d}\right)$. Corollary 3.3 is proved.

The previous theorem implies the Slutsky theorem for the LPD in $l_{\infty}(T)$. Under the conditions in the previous theorem, by the contraction principle for any continuous function $g$ in $\mathbf{R}^{d},\left\{g\left(U_{n}^{(1)}(t), \ldots, U_{n}^{(d)}(t)\right): t \in T\right\}$ satisfies the LDP in $l_{\infty}(T)$ with speed $\varepsilon_{n}^{-1}$.

The next corollary gives necessary and sufficient conditions for the LDP for Banach space valued r.v.'s.

Corollary 3.4. Let $\left\{X_{n}\right\}_{n=1}^{\infty}$ be a sequence of r.v.'s with values in Banach space $B$. Then $\left\{X_{n}\right\}_{n=1}^{\infty}$ satisfies the LDP in $B$ with speed $\varepsilon_{n}^{-1}$ if and only if for each $f_{1}, \ldots, f_{m} \in B^{*},\left(f_{1}\left(X_{n}\right), \ldots, f_{m}\left(X_{n}\right)\right)$ satisfies the LDP with speed $\varepsilon_{n}^{-1}$ and rate function $I_{f_{1}, \ldots, f_{m}}$, and for each $\tau>0$,

$$
\lim _{\eta \rightarrow 0} \limsup _{n \rightarrow \infty} \varepsilon_{n} \ln \left(\mathbf{P}^{*}\left\{\sup _{f_{1}, f_{2} \in B_{1}^{*}, \rho\left(f_{1}, f_{2}\right) \leqslant \eta}\left|f_{1}\left(X_{n}\right)-f_{2}\left(X_{n}\right)\right| \geqslant \tau\right\}\right)=-\infty,
$$

where $\rho$ is as in (3.3).

P r o o f. Consider $\phi: B \rightarrow l_{\infty}\left(B_{1}^{*}\right)$, defined by $\phi(z)=\left\{f(z): f \in B_{1}^{*}\right\}$. It is easy to see that $\phi: B \rightarrow \phi(B)$ is a continuous one-to-one function with continuous inverse. Thus, by the contraction principle, $X_{n}$ satisfies the LDP in $B$ if and only if $\left\{f\left(X_{n}\right): f \in B_{1}^{*}\right\}$ satisfy the LDP in $l_{\infty}(T)$. Theorem 3.1 implies the claim. Corollary 3.4 is proved.

Next, we consider the LDP for stochastic processes whose sample paths are a convex function on the parameter. It is well known that if a sequence of convex functions converges, then the convergence is uniformly on a compact set (see Theorem 10.8 in [27]). A similar result holds for the weak convergence of stochastic processes (see [2]). We will use some of the techniques 
in this paper. In particular Lemma 13 in [2] says that for each convex function $f:[-1,1]^{d} \rightarrow \mathbf{R}$,

$$
\sup _{x \in[-1,1]^{d}}|f(x)| \leqslant 3^{d} \sup _{x \in\{-1,0,1\}^{d}}|f(x)| .
$$

We also will need the following fact (see Lemma 14 in [2]): let $T_{0}$ be a set of $\mathbf{R}^{d}$, let $\varepsilon>0$, let $T_{0}^{\varepsilon}=\left\{x+y: x \in T_{0},|y| \leqslant \varepsilon\right\}$ and let $f: T_{0}^{\varepsilon} \rightarrow \mathbf{R}$ be a convex function, then for each $x, y \in T_{0}$

$$
|f(x)-f(y)| \leqslant|x-y| \varepsilon^{-1} 2 \sup _{t \in T_{0}^{\varepsilon}}|f(t)| .
$$

Corollary 3.5. Let $T_{0}$ be an open convex set of $\mathbf{R}^{d}$. Let $T$ be a compact set of $T_{0}$. Let $\left\{\varepsilon_{n}\right\}$ be a sequence of positive numbers converging to zero. Let $\left\{U_{n}(t): t \in T_{0}\right\}$ be a sequence of stochastic processes. Suppose that:

(i) $U_{n}(t)$ is a convex function in $t$;

(ii) for each $t_{1}, \ldots, t_{m} \in T_{0},\left(U_{n}\left(t_{1}\right), \ldots, U_{n}\left(t_{m}\right)\right)$ satisfies the $L D P$ with speed $\varepsilon_{n}^{-1}$.

Then $\left\{U_{n}(t): t \in T\right\}$ satisfies the LDP in $l_{\infty}(T)$ with speed $\varepsilon_{n}^{-1}$.

$\mathrm{P}$ r o of. We have to prove that given $c, \eta>0$, there exists a finite partition function $\pi$ of $T_{0}$ such that

$$
\limsup _{n \rightarrow \infty} \varepsilon_{n} \ln \left(\mathbf{P}^{*}\left\{\sup _{t \in T_{0}}\left|U_{n}(t)-U_{n}(\pi(t))\right| \geqslant \eta\right\}\right) \leqslant-c .
$$

Take $\varepsilon>0$ such that $T_{0}^{\varepsilon} \subset T$. Since $T_{0}^{\varepsilon}$ is a compact set, it can be covered by a finite number of hypercubes. So, by (3.18), there are $t_{1}, \ldots, t_{m} \in T$ such that for each convex function $h$ defined on $T, \sup _{t \in T_{0}^{\varepsilon}}|h(t)| \leqslant$ $3^{d} \max _{1 \leqslant l \leqslant m}\left|h\left(t_{l}\right)\right|$. Hence there exists a finite constant $M$ such that

$$
\lim _{n \rightarrow \infty} \varepsilon_{n} \ln \left(\mathbf{P}\left\{\max _{1 \leqslant l \leqslant m}\left|U_{n}\left(t_{l}\right)\right| \geqslant M\right\}\right) \leqslant-c .
$$

Take a finite partition function $\pi$ of $T_{0}$ such that $\sup _{t \in T_{0}}|t-\pi(t)| \leqslant$ $2^{-1} M^{-1} 3^{-d} \eta \varepsilon$. By (3.19),

$$
\sup _{t \in T_{0}}\left|U_{n}(t)-U_{n}(\pi(t))\right| \leqslant M^{-1} 3^{-d} \eta \sup _{t \in T_{0}^{\delta}}\left|U_{n}(t)\right| .
$$

Therefore,

$$
\begin{aligned}
& \limsup _{n \rightarrow \infty} \varepsilon_{n} \ln \left(\mathbf{P}^{*}\left\{\sup _{t \in T_{0}}\left|U_{n}(t)-U_{n}(\pi(t))\right| \geqslant \eta\right\}\right) \\
& \leqslant \limsup _{n \rightarrow \infty} \varepsilon_{n} \ln \left(\mathbf{P}^{*}\left\{\sup _{t \in T_{0}^{\varepsilon}}\left|U_{n}(t)\right| \geqslant M 3^{d}\right\}\right) \\
& \leqslant \limsup _{n \rightarrow \infty} \varepsilon_{n} \ln \left(\mathbf{P}\left\{\max _{1 \leqslant l \leqslant m}\left|U_{n}\left(t_{l}\right)\right| \geqslant M\right\}\right) \leqslant-c .
\end{aligned}
$$

Hence the claim follows. Corollary 3.5 is proved.

Next, we consider the case of nondecreasing processes. 
Corollary 3.6. Let $\left\{U_{n}(t): 0 \leqslant t \leqslant M\right\}$ be a sequence of stochastic processes. Let $\left\{\varepsilon_{n}\right\}$ be a sequence of positive numbers converging to zero. Suppose that:

(i) With probability one, $U_{n}(t)$ is a nondecreasing function in $t$;

(ii) for each $0 \leqslant t_{1}<\cdots<t_{m} \leqslant M,\left(U_{n}\left(t_{1}\right), \ldots, U_{n}\left(t_{m}\right)\right)$ satisfies the $L D P$ with speed $\varepsilon_{n}^{-1}$;

(iii) for each $0<k<\infty$ and each $0 \leqslant t_{0} \leqslant M$,

$$
\lim _{t \rightarrow t_{0}} \sup \left\{\left|u_{2}-u_{1}\right|: I_{t, t_{0}}\left(u_{1}, u_{2}\right) \mid \leqslant k\right\}=0,
$$

where $I_{t, t_{0}}$ is the rate function of the LDP for $\left(U_{n}(t), U_{n}\left(t_{0}\right)\right)$.

Then $\left\{U_{n}(t): 0 \leqslant t \leqslant M\right\}$ satisfies the LDP in $l_{\infty}([0, M])$ with speed $\varepsilon_{n}^{-1}$.

P r o o f. By an argument in the proof of Corollary 3.1, condition (ii) implies that for each $0<k<\infty$,

$$
\lim _{\eta \rightarrow 0} \sup _{0 \leqslant s, t \leqslant M,|s-t| \leqslant \eta} \sup \left\{\left|u_{2}-u_{1}\right|: I_{s, t}\left(u_{1}, u_{2}\right) \mid \leqslant k\right\}=0 .
$$

First, we prove that for each $\tau>0$,

$$
\lim _{\eta \rightarrow 0} \inf \left\{I_{s, t}\left(u_{1}, u_{2}\right):\left|u_{2}-u_{1}\right| \geqslant \tau, 0 \leqslant s, t \leqslant M,|s-t| \leqslant \eta\right\}=\infty .
$$

Given $0<k<\infty$ and $\tau>0$, there exists an $\eta>0$ such that

$$
\sup _{0 \leqslant s, t \leqslant M,|s-t| \leqslant \eta} \sup \left\{\left|u_{2}-u_{1}\right|: I_{s, t}\left(u_{1}, u_{2}\right) \mid \leqslant k\right\}<\tau .
$$

Hence, $\inf \left\{I_{s, t}\left(u_{1}, u_{2}\right):\left|u_{2}-u_{1}\right| \geqslant \tau, 0 \leqslant s, t \leqslant M,|s-t| \leqslant \eta\right\} \geqslant k$, and (3.20) holds.

Given a positive integer $m$, we have that

$$
\begin{aligned}
& \mathbf{P}\left\{\max _{1 \leqslant i \leqslant m} \sup _{t_{i-1} \leqslant t \leqslant t_{i}}\left|U_{n}(t)-U_{n}\left(t_{i-1}\right)\right| \geqslant \tau\right\} \\
& \quad \leqslant \max _{1 \leqslant i \leqslant m} \mathbf{P}\left\{U_{n}\left(t_{i}\right)-U_{n}\left(t_{i-1}\right) \geqslant \tau\right\},
\end{aligned}
$$

where $t_{i}=m^{-1} M i$. So,

$$
\begin{aligned}
& \limsup _{n \rightarrow \infty} \varepsilon_{n} \ln \left(\mathbf{P}\left\{\max _{1 \leqslant i \leqslant m} \sup _{t_{i-1} \leqslant t \leqslant t_{i}}\left|U_{n}(t)-U_{n}\left(t_{i-1}\right)\right| \geqslant \tau\right\}\right) \\
& \leqslant-\inf \left\{I_{t_{i-1}, t_{i}}(u, v):|v-u| \geqslant \tau, 1 \leqslant i \leqslant m\right\},
\end{aligned}
$$

which tends to $\infty$, as $m \rightarrow \infty$. Corollary 3.6 is proved.

In the previous theorem, conditions (i) and (ii), without condition (iii), are not sufficient to obtain the thesis of theorem. In the example considered in Theorem 6.2 (see Part II) when $\mu[0, x]$ is slowly varying at infinity, conditions (i) and (ii) in the previous theorem hold, but the stochastic process 
does not satisfy the LDP. In other words, the LDP for the finite dimensional distributions of a nondecreasing stochastic process does not imply the uniform LDP.

We will need the following proposition, whose proof is omitted because it is trivial.

Theorem 3.2. Let $\left\{U_{n}(t): t \in \mathbf{R}\right\}$ be a sequence of stochastic processes. Suppose that for each $0<M<\infty,\left\{U_{n}(t):|t| \leqslant M\right\}$ satisfies the $L D P$ in $l_{\infty}[-M, M]$ with speed $\varepsilon_{n}^{-1}$ and rate function $I_{M}$.

Then $\left\{U_{n}(t): t \in \mathbf{R}\right\}$ satisfies the LDP in $\left(\mathscr{F}(\mathbf{R}), d_{\text {comp }}\right)$ with speed $\varepsilon_{n}^{-1}$ and rate function $I$, where

$$
\begin{gathered}
\mathscr{F}(\mathbf{R})=\left\{\alpha: \mathbf{R} \rightarrow \mathbf{R}: \sup _{|t| \leqslant M}|\alpha(t)|<\infty, \text { for each } M<\infty\right\} \\
d_{\text {comp }}(\alpha, \beta)=\sum_{k=1}^{\infty} \frac{|\alpha-\beta|_{l_{\infty}[-k, k]} \wedge 1}{2^{k}}, \quad I(z)=\lim _{M \rightarrow \infty} I_{M}\left(\left.z\right|_{[-M, M]}\right)
\end{gathered}
$$

and $\left.z\right|_{[-M, M]}$ is $z$ restricted to $[-M, M]$.

Next, we consider the compositions of stochastic processes.

Theorem 3.3. Let $\left\{U_{n}(t): t \in \mathbf{R}\right\}$ and $\left\{V_{n}(t): 0 \leqslant t \leqslant M_{2}\right\}$ be two sequences of stochastic processes. Suppose that:

(i) For each $M_{1}<\infty$,

$$
\left\{U_{n}(t):|t| \leqslant M_{1}\right\} \times\left\{V_{n}(t): 0 \leqslant t \leqslant M_{2}\right\}
$$

satisfies the LDP in $l_{\infty}\left(\left[-M_{1}, M_{1}\right]\right) \times l_{\infty}\left(\left[0, M_{2}\right]\right)$ with speed $\varepsilon_{n}^{-1}$ and rate function $I_{M_{1}}^{(U, V)}$;

(ii) for each $t \in \mathbf{R}$ and each positive integer $k$

$$
\lim _{t \rightarrow t_{0}} \sup \left\{\left|u_{2}-u_{1}\right|: I_{t, t_{0}}^{(U)}\left(u_{1}, u_{2}\right) \leqslant k\right\}=0,
$$

where $I_{t, t_{0}}^{(U)}$ is the rate function of the LDP for $\left(U_{n}(t), U_{n}\left(t_{0}\right)\right)$.

Then $\left\{U_{n}\left(V_{n}(t)\right): 0 \leqslant t \leqslant M_{2}\right\}$ satisfies the LDP in $l_{\infty}\left(\left[0, M_{2}\right]\right)$ with speed $\varepsilon_{n}^{-1}$. Moreover, the rate function is

$$
I(z)=\inf \left\{I^{(U, V)}(\alpha, \beta): \alpha \circ \beta=z\right\},
$$

where $I^{(U, V)}(\alpha, \beta)=\lim _{M_{1} \rightarrow \infty} I_{M_{1}}^{(U, V)}\left(\left.\alpha\right|_{\left[-M_{1}, M_{1}\right]}, \beta\right)$.

P r o o f. By Theorem 3.2, we have that $\left(U_{n}, V_{n}\right)$ satisfies the LDP in $\left(\mathscr{F}(\mathbf{R}), d_{\text {comp }}\right) \times l_{\infty}\left[-M_{2}, M_{2}\right]$ with speed $\varepsilon_{n}^{-1}$ and rate function $I^{(U, V)}$. Let $\phi:\left(\mathscr{F}(\mathbf{R}), d_{\text {comp }}\right) \times l_{\infty}\left[-M_{2}, M_{2}\right] \rightarrow l_{\infty}\left[-M_{2}, M_{2}\right]$ be defined by $\phi(\alpha, \beta)=$ $\alpha \circ \beta$. By condition (ii), if $I^{(U, V)}(\alpha, \beta)<\infty$, then $\alpha$ is continuous. Hence, $\phi$ is continuous at each $(\alpha, \beta)$ with $I^{(U, V)}(\alpha, \beta)<\infty$. We claim that $\varphi$ is continuous at each $(\alpha, \beta)$ with $I^{(U, V)}(\alpha, \beta)<\infty$. Observe that if $\left(\alpha_{n}, \beta_{n}\right) \rightarrow(\alpha, \beta)$ in $\mathscr{F}(\mathbf{R}) \times l_{\infty}\left[-M_{2}, M_{2}\right]$, then $M_{1}:=\sup _{n \geqslant 1} \sup _{|t| \leqslant M_{2}}\left|\beta_{n}(t)\right|<\infty$. Since 
$\alpha_{n} \rightarrow \alpha$ in $l_{\infty}\left[-M_{2}, M_{2}\right], \beta_{n} \rightarrow \beta$ in $l_{\infty}\left[-M_{1}, M_{1}\right]$ and $\alpha$ is uniformly continuous on $l_{\infty}\left[-M_{1}, M_{1}\right]$,

$$
\begin{aligned}
& \sup _{|t| \leqslant M_{2}}\left|\alpha_{n}\left(\beta_{n}(t)\right)-\alpha(\beta(t))\right| \\
& \quad \leqslant \sup _{|t| \leqslant M_{2}}\left|\alpha_{n}\left(\beta_{n}(t)\right)-\alpha\left(\beta_{n}(t)\right)\right|+\sup _{|t| \leqslant M_{2}}\left|\alpha\left(\beta_{n}(t)\right)-\alpha(\beta(t))\right| \\
& \quad \leqslant \sup _{|t| \leqslant M_{1}}\left|\alpha_{n}(t)-\alpha(t)\right|+\sup _{|t| \leqslant M_{2}}\left|\alpha\left(\beta_{n}(t)\right)-\alpha(\beta(t))\right| \longrightarrow 0 .
\end{aligned}
$$

Hence, by Corollary 2.1, $\left\{U_{n} \circ V_{n}\right\}$ satisfies the LDP in $l_{\infty}\left[-M_{2}, M_{2}\right]$ with speed $\varepsilon_{n}^{-1}$. Theorem 3.3 is proved.

There are variations of the previous theorem which hold in an obvious way. For example, we may consider the processes $\left\{U_{n}(t): t \geqslant 0\right\}$ and $\left\{V_{n}(t): 0 \leqslant t \leqslant M_{2}\right\}$. Under the conditions in the previous theorem, we obtain the LDP for $\left\{U_{n}\left(\left|V_{n}(t)\right|\right): 0 \leqslant t \leqslant M_{2}\right\}$.

\section{REFERENCES}

1. Arcones M. A. On the law of the iterated logarithm for Gaussian Processes. - J. Theoret. Probab., 1995, v. 8, № 4, p. 877-903.

2. Arcones M. A. Weak convergence of convex stochastic processes. - Statist. Probab. Lett., 1998, v. 37, № 2, p. 171-182.

3. Bahadur R. R. Some Limit Theorems in Statistics. Philadelphia, PA: SIAM, 1971, $42 \mathrm{p}$.

4. Bingham N. H., Goldie C. M., Teugels J. L. Regular Variation. Cambridge: Cambridge Univ. Press, 1987, 491 p.

5. Borell C. The Brunn-Minkowski inequality in Gauss space. - Invent. Math., 1975, v. 30 , p. $207-216$.

6. Burdzy K. Some path properties of iterated Brownian motion. - Seminar on Stochastic Processes, 1992 (Seattle, WA, 1992). Boston: Birkhäuser, 1993, p. 67-87. (Progr. Probab., v. 33.)

7. Burdzy K. Variation of iterated Brownian motion. -- Measure-Valued Processes, Stochastic Partial Differential Equations, and Interacting Systems (Montreal, PQ, 1992). Providence, RI: Amer. Math. Soc., 1994, p. 35-53. (CRM Proc. Lecture Notes, v. 5.)

8. Chevet $S$. Gaussian measures and large deviations. - Probability in Banach Spaces, IV (Oberwolfach, 1982). Berlin-Heidelberg: Springer-Verlag, 1983, p. 30-46. (Lecture Notes in Math., v. 990.)

9. Csáki E., Csörgö M., Földes A., Révész P. Global Strassen-type theorems for iterated Brownian motions. - Stochastic Process. Appl., 1995, v. 59, № 2, p. 321-341.

10. Csáki E., Földes A., Révész P. Strassen theorems for a class of iterated processes. Trans. Amer. Math. Soc., 1997, v. 349, № 3, p. 1153-1167.

11. Deheuvels P., Mason D. M. A functional L.I.L. approach to pointwise Bahadur-Kiefer theorems. - Probability in Banach Spaces 8: Proceedings of the Eighth international conference. Ed. by R. M. Dudley et al. Boston: Birkhäuser, 1992, p. 255-266.

12. Dembo A., Zeitouni O. Large Deviations Techniques and Applications. New York: Springer-Verlag, 1998, $396 \mathrm{p}$.

13. Deuschel J.-D., Stroock D. W. Large Deviations. Boston, MA: Academic Press, 1989, $307 \mathrm{p}$. 
14. Donsker M.D., Varadhan S. R.S. Asymptotic evaluation of certain Markov process expectations for large time. III. - Comm. Pure Appl. Math., 1976, v. 29, № 4, p. 389461.

15. Dudley R. M. Uniform Central Limit Theorems. Cambridge: Cambridge Univ. Press, 1999, $436 \mathrm{p}$.

16. Dunford N., Schwartz J.T. Linear Operators. Part I: General Theory. New York: Wiley, 1988, $858 \mathrm{p}$.

17. Ellis R. S. Large deviations for a general class of random vectors. - Ann. Probab., 1984, v. 12 , № 1, p. 1-12.

18. Funaki T. Probabilistic construction of the solution of some higher order parabolic differential equations. - Proc. Japan Acad., 1979, v. 55, № 5, p. 176-179.

19. Hoffmann-Jørgensen J. Stochastic Processes on Polish Spaces. Aarhus: Aarhus Univ., 1991, 278 p. (Various Publications Ser., № 39.)

20. $\mathrm{Hu} Y$., Shi $Z$. The Csörgö-Révész modulus of non-differentiability of iterated Brownian motion. - Stochastic Process. Appl., 1995, v. 58, № 2, p. 267-279.

21. Hu Y., Pierre-Loti-Viaud D., Shi Z. Laws of the iterated logarithm for iterated Wiener processes. - J. Theoret. Probab., 1995, v. 8, № 2, p. 303-319.

22. Khoshnevisan D., Lewis T. M. The uniform modulus of continuity of iterated Brownian motion. - J. Theoret. Probab., 1996, v. 9, № 2, p. 317-333.

23. Khoshnevisan D., Lewis T. M. Chung's law of the iterated logarithm for iterated Brownian motion. - Ann. Inst. H. Poincaré Probab. Statist., 1996, v. 32, № 3, p. 349359 .

24. Lynch J., Sethuraman J. Large deviations for processes with independent increments. - Ann. Probab., 1987, v. 15, № 2, p. 610-627.

25. Puhalskii A. On functional principle of large deviations. - New Trends in Probability and Statistics (Bakuriani, 1990). V. 1. Utrecht: VSP, 1991, p. 198-218.

26. Rao M. M., Ren Z. D. Theory of Orlicz Spaces. New York: Dekker, 1991, 449 p.

27. Рокафеллар Р. Выпуклый анализ. М.: Мир, 1973, 469 с.

28. Schilder $M$. Some asymptotic formulas for Wiener integrals. - Trans. Amer. Math. Soc., 1966, v. 134, p. 193-216.

29. Shi $Z$. Lower limits of iterated Wiener processes. - Statist. Probab. Lett., 1995, v. 23, № 3, p. 259-270.

30. Судаков В.Н., Цирельсон Б. С. Экстремальные свойства полупространств для сферически инвариантных мер. - Зап. научн. семин. ЛОМИ, 1974, т. 41, с. 1424.

31. van der Vaart A. W., Wellner J. A. Weak Convergence and Empirical Processes. With Applications to Statistics. New York: Springer-Verlag, 1996, 508 p.

32. Varadhan S. R. S. Asymptotic probabilities and differential equations. - Comm. Pure Appl. Math., 1966, v. 19, p. 261-286.

33. Varadhan S. R. S. Large Deviations and Applications. Philadelphia: SIAM, 1984, 75 p.

34. Willard S. General Topology. Reading: Addison-Wesley, 1970, 369 p.

Поступила в редакцию 5.IV.2001 